\title{
Tecnología lítica y petroglifos en Barrancas, Jujuy
}

\section{( Patricio Kohan*}

\author{
Fecha de defensa: 6 de noviembre de 2018 \\ Director: Dr. Rodolphe Hoguin \\ Jurados: Dres. Federico Restifo y Hugo \\ Yacobaccio
}

\section{Introducción}

La presente tesis de licenciatura se propuso analizar la relación entre el arte rupestre y la tecnología lítica a partir del estudio de dos sitios arqueológicos: Laguna Media 7 y Morro Blanco. En la actualidad, dicha asociación entre tecnología lítica y arte rupestre ha comenzado a ser explorada, lo que a su vez ha permitido comprender con un mayor grado de resolución los procesos tecnológicos detrás de la manufactura de pinturas, petroglifos y grabados así como también los instrumentos necesarios para su elaboración (Alvarez, Fiore, Favret, y Guerra, 2001; Blanco y Lynch, 2011; Santos Da Rosa, Cura, Garces, y Cura, 2014; Yacobaccio, Catá, Solá, y Alonso, 2008). A pesar de que tradicionalmente la tecnología lítica fue considerada como una línea de evidencia diametralmente opuesta a la del arte, planteándoselas como dos esferas independientes, la primera como un objeto material que responde a necesidades funcionales (Fiore, 2014) y la segunda como posible de abordarse desde la expresión simbólica (Ingold, 1999), se ha demostrado que es posible complementar una con otra, analizando la relación entre ambas y superando una supuesta dicotomía de "mente vs. materia". Es necesario sin embargo, contar con un marco teórico y una metodología que se adecue al caso de estudio y los objetivos puntuales a cumplir. En los análisis aquí realizados, el foco estuvo puesto en la identificación de potenciales instrumentos líticos que hayan cumplido la función de grabado para diversos motivos y petroglifos en el sitio de Morro Blanco.

\section{Sitios y área de estudio}

Morro Blanco y Laguna Media 7 se encuentran en las proximidades de la localidad de Abdón Castro Tolay (conocido también como Barrancas), departamento de Cochinoca, provincia de Jujuy. El área circundante al pueblo de Barrancas posee un gran potencial arqueológico, encontrándose más de 40 sitios arqueológicos en las proximidades del río homónimo, presentando una marcada asociación con representaciones de arte rupestre en los mismos. Los sitios de Laguna Media 7 y Morro Blanco

* Instituto de Arqueología, Facultad de Filosofía y Letras, Universidad de Buenos Aires (UBA). 25 de mayo 217, 3 o piso (CP C1002ABE) Ciudad Autónoma de Buenos Aires, Argentina. E-mail:patriciokohan.91@gmail.com fueron ocupados por poblaciones humanas en plena transición entre Holoceno medio y tardío, momento en el cual las economías basadas en la caza y recolección comienzan a sufrir transformaciones que culminan en sistemas económicos pastoriles durante el Holoceno tardío. Mientras que la ocupación en Laguna Media 7 corresponde a un rango de entre 3562-3703 años cal. a.C., Morro Blanco posee 3 fechados: 3144-3349 años cal. a.C. en la zona del refugio del alero, y 2676-2742 años cal. a.C. y 2489-2644 años cal. a.C. en el sector de las terrazas del sitio. La temporalidad de estos sitios y su contexto de cambio y transformación influye en las diversas esferas de la acción humana encontrándose la tecnología entre ellas, viéndose así reflejados los conjuntos líticos correspondientes.

Por otro lado, el ambiente, fauna y flora de la región son característicos de la Puna de Jujuy, distinguiéndose un clima típico de desierto de altura dado que ambos sitios se encuentran por arriba de los 3.600 metros sobre el nivel del mar. Analizándose a mayor resolución espacial y temporal y a partir de análisis paleoambientales basados en el perfil Cruces 2 ( $P C C_{2}$ ) se determinó que a pesar de seguir con las tendencias generales de la transición entre Holoceno medio y tardío, existió una mayor variabilidad ambiental a nivel local (Pirola et al., 2017; Yacobaccio y Morales, 2011). Las condiciones ambientales pasaron de ser más secas y estables hacia un aumento de la humedad e inestabilidad, influyendo asimismo el sistema hidrológico de Barrancas.

\section{Problema arqueológico}

El problema arqueológico tratado apuntó a llenar un vacío en el conocimiento actual del área, referido al proceso de confección de petroglifos, partiendo de los instrumentos grabadores como el elemento clave para su ejecución. Para ello, se complementaron dos líneas de evidencia distintas, el arte rupestre (Figura 1) y la tecnología lítica (Figura 2), lo que permitió caracterizar la relación tecnológica entre el conjunto lítico y el arte rupestre. Para ello, primero se identificaron las materias primas presentes en los conjuntos líticos, evaluando su potencial para la ejecución de actividades de grabado y distinguiendo si hubo un proceso de selección de materiales. Luego se analizaron los conjuntos líticos de Laguna Media 7 y Morro Blanco identificando y caracterizando los potenciales instrumentos involucrados en la confección de petroglifos a partir de sus Unidades Tecno Funcionales (UTF), y finalmente se identificaron los rastros de uso sobre dichas UTF Transformativas, permitiendo establecer los patrones resultantes del trabajo sobre distintas materias primas y por consiguiente determinar su funcionalidad artefactual. 


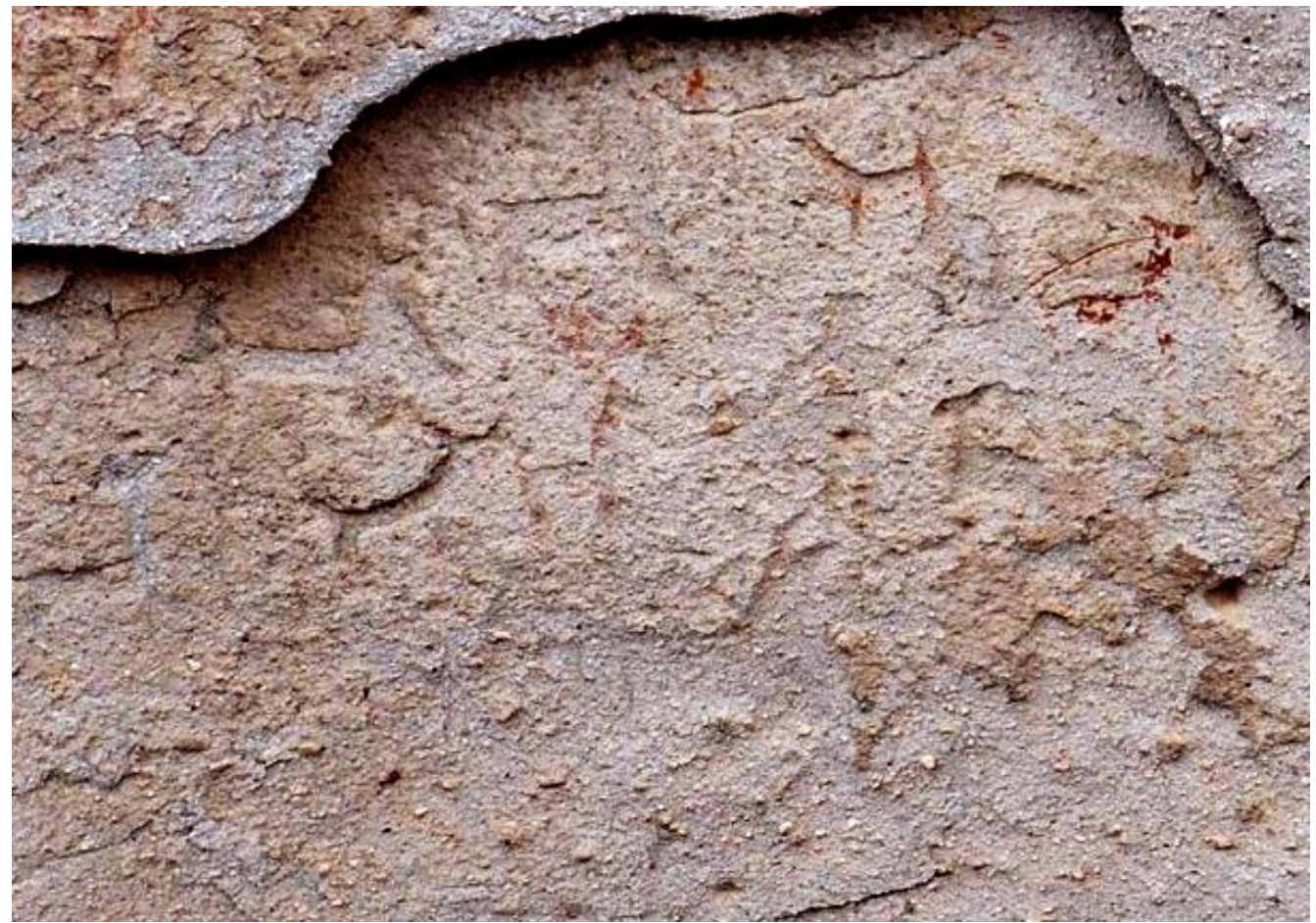

Figura 1. Soporte rocoso con petroglifos en Morro Blanco.
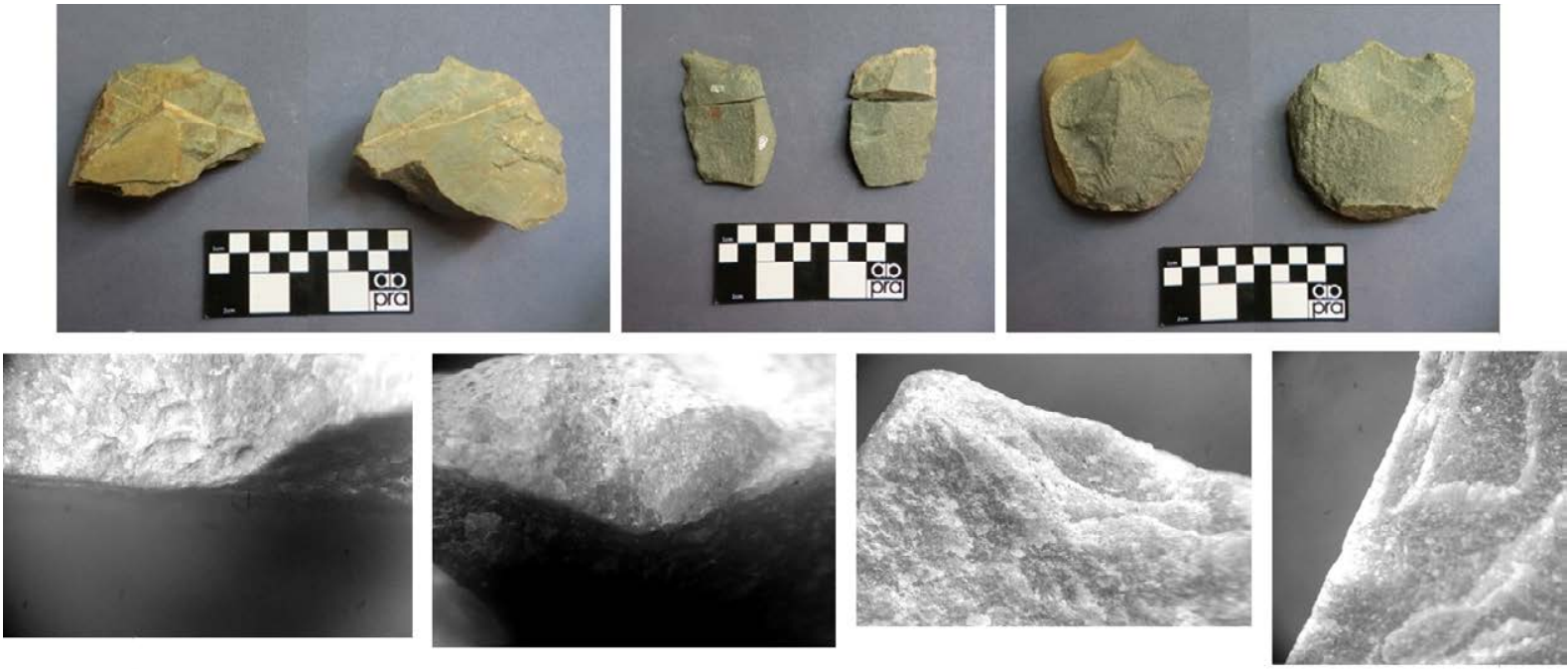

Figura 2. Instrumentos grabadores (fila superior) y sus rastros de uso en las zonas de contacto de las UTF transformativas (fila inferior).

\section{Marco teórico}

El marco teórico en el cual se desarrolló la presente tesis fue el de la Antropología de los Sistemas Tecnológicos (Lemonnier, 1986, 1992), ya que el mismo permite contemplar a la evidencia lítica como un elemento conformado por diversas interacciones a nivel social entre humanos, medio y tecnología, siendo la misma parte de un sistema más amplio, incluyendo el análisis de las técnicas propias del mismo. A modo de complemento con el marco teórico de Lemonnier y con el objetivo de ahondar aún más en el comportamiento tecnológico de los grupos humanos que ocuparon los sitios, los instrumentos grabadores fueron analizados en relación a su organización tecnológica (Nelson, 1991). Finalmente, se utilizaron dos elementos centrales para la implementación del marco teórico propuesto por Lemonnier: las cadenas operativas y los análisis tecnofuncionales, ambos conceptos elaborados y propuestos por Eric Boëda (Boëda, 2013; Boëda, Geneste 
y Meignen, 1998), lo que permitió seleccionar la muestra sobre la cual se realizaron los análisis funcionales de rastros de uso correspondientes (Mansur, 1999; Tringham, Cooper, Odell, Voytek y Whitman, 1974).

\section{Resultados}

Como ya se mencionó previamente, los análisis constaron de tres partes: la identificación de las materias primas, los análisis tecnofuncionales sobre los conjuntos tecnológicos y los análisis de rastros de uso sobre los instrumentos grabadores. Para comenzar, se pudo identificar que la composición de la mayoría de los instrumentos grabadores fue de cuarcita de origen local y fácil acceso. Sin embargo, al analizar las propiedades físicas y mecánicas de todas las materias primas presentes en el conjunto y la disponibilidad y acceso a ellas, se reveló un proceso de selección de la cuarcita dado el tamaño de sus nódulos y sus propiedades físicas, aptas para realizar actividades de grabado, inciso o raspado sobre los soportes duros y abrasivos de Morro Blanco.

En segundo lugar, los análisis tecnológicos y de UTF entre ambos sitios mostraron una relación interesante. Tecnológicamente, ambos sitios poseen esquemas de desbaste y façonnage similares, distinguiéndose en las UTF representadas y sus proporciones. Esto podría deberse no solo a una funcionalidad diferencial entre los sitios, sino también a su distancia temporal representada en este momento de transición, donde la utilización de sistemas y modos de talla se perpetúan en el tiempo, modificando algunos aspectos a partir de las decisiones individuales tomadas por los talladores. Aquí se lograron identificar aquellos instrumentos con las UTF Transformativas que poseían mayor potencial para la elaboración de grabados.

Finalmente, sobre estos instrumentos potencialmente grabadores, al realizar los análisis de rastros de uso utilizando bajos aumentos e identificando la presencia, posición y morfología de microrastros, esquirlamientos y estrías, se logró corroborar que los instrumentos con UTF de buril y filo sinuoso de Morro Blanco cumplieron funciones asociadas a la elaboración de petroglifos. Esto fue posible gracias a la elaboración de un marco de referencia bibliográfico, realizado a partir del relevamiento de rastros comúnmente asociados con el trabajo sobre soportes rocosos de materias primas heterogéneas como la cuarcita (Adams et al., 2009; Alvarez y Fiore, 1995; Blanco y Lynch, 2011; Clemente-Conte y Gibaja, 2009; Leipus y Mansur, 2007; Mansur, 1999; Marreiros, Mazzucco, Gibaja y Bicho, 2015; Santos da Rosa, 2012).

Lo relevante de los análisis radica en su carácter complementario, ya que la información provista individualmente no sería suficiente para arribar a conclusiones claras sobre el proceso de manufactura de los petroglifos en el sitio de Morro Blanco. Se demuestra por lo tanto la utilidad de abordar un problema arqueológico desde distintas líneas de evidencia, utilizando marcos teóricos y metodológicos flexibles, y diversas líneas de análisis con el objetivo de obtener una visión más holística y completa de aquellos procesos pasados a estudiar.

\section{Referencias citadas}

»Adams, J., Delgado, S., Dubreuil, L., Hamon, C., Plisson, H. y Risch, R. (2009). Functional analysis of macro-lithic artefacts: A focus on working surfaces. En F. Sternke, L. Eigeland y C. Laurent-Jacques (Eds.), Non-flint raw material use in Prehistory. Old prejudices and new directions (pp. 43-66). Oxford: BAR International Series.

» Alvarez, M. y Fiore, D. (1995). Recreando imagenes: Diseño de experimentacion acerca de las tecnicas y los artefactos para realizar grabados de arte rupestre. Cuadernos del Instituto Nacional de Antropologia y Pensamiento Latinoamericano, 16, 215-239.

» Alvarez, M. Fiore, D., Favret, E. y Guerra, R. C. (2001). The use of lithic artefacts for making rock art engravings: observation and analysis of use-wear traces in experimental tools through optical microscopy and SEM. Journal of Archaeological Science, 28, 457-464.

» Blanco, R. y Lynch, V. (2011). Implicancias en el arte rupestre de la localidad arqueológica de Piedra Museo (Santa Cruz, Argentina). Boletín Del Museo Chileno de Arte Precolombino, 16, 9-21.

» Boëda, E. (2013). Techno-logique \& Technologie: Une paléo-histoire des objets lithiques tranchants. (1ra ed.). Paris: Archeo-editions.com.

» Boëda, E., Geneste, J. y Meignen, L. (1998). Identification de chaines operatoires lithiques du Paleolithique ancien et moyen. Paleo, 2, 43-80.

"Clemente-Conte, I. y Gibaja, J. F. (2009). Formation of use-wear traces in non-flint rocks: The case of quartzite and rhyolite- Differences and similarities. En F. Sternke, L. Eigeland y L.-J. Costa (Eds.), International union por Prehistoric and Protohistoric sciences. NonFlint Raw Material Use in Prehistory Old prejudices and new directions (pp. 4-9). Oxford: British Archaeological Reports.

»Fiore, D. (2014). Arqueología del arte: Marcos teóricos. En C. Smith (Ed.), Archaeology of Art: Theoretical Frameworks. Encyclopedia of Global Archaeology (pp. 436-449). Nueva York: Springer.

» Ingold, T. (1999). Afterword. En M. A. Dobres y C. Hoffman (Eds.), The social dynamics of technology. Washington: Smithsonian Institute Press. 
»Leipus, M. y Mansur, M. E. (2007). El análisis funcional de base microscópica aplicado a materiales heterogéneos. Perspectivas metodológicas para el estudio de las cuarcitas de la región pampeana. En En C. Bayón, A. Pupio, M. I. González, N. Flegenheimer y M. Frère (Eds.), Arqueología en las Pampas (Tomo I) (pp. 179-200). Buenos Aires: Sociedad Argentina de Antropología.

»Lemonnier, P. (1986). The study of material culture today: Toward an anthropology of technical systems. Journal of Anthropological Archaeology, 5, 147-186.

"Lemonnier, P. (1992). Elements for an Anthropology of Technology. Anthropological Papers, Museum of Anthropology, University of Michigan, 88, 1-24.

» Mansur, M. E. (1999). Análisis funcional de instrumental lítico: problemas de formación y deformación de rastros de uso. En Actas del XII Congreso Nacional de Arqueología Argentina (pp. 355-366). La Plata.

" Marreiros, J., Mazzucco, N., Gibaja, J. F. y Bicho, N. (2015). Macro and micro evidence from the past: The state of the art of archaeological use-wear studies. En Use-wear and residue analysis in Archaeology (pp. 5-26). Springer: USA

» Nelson, M. (1991). The study oftechnological organization. Archaeological Method and Theory, 3, 57-100.

» Pirola, M., Bustos, S., Morales, M., Orgeira, J., Oxman, B., Tchilinguirian, P. y Vázquez, C. (2017). The Mid to
Late Holocene transition in Barrancas, Jujuy, Argentina: Regional climate change, local environments and archaeological implications. Journal of Archaeological Science: Reports, 6, 1-17.

"Santos da Rosa, N. (2012). Contribuição para o estudo do Vale do Tejo (Portugal): O sítio Cachão do Algarve. Universidade de Trás-os-Montes e Alto Douro.

"Santos Da Rosa, N., Cura, S., Garces, S. y Cura, P. (2014). Between tools and engravings: Technology and experimental archaeology to the study of Cachão de Algarve Rock Art. En S. Cura, J. Cerezer, M. Gurova, B. Santander, L. Oosterbeek y C. Jorge (Eds.), Union Internationale des Sciences Prèhistoriques et Protohistoriques (pp. 87-96). Oxford: British Archaeological Reports.

» Tringham, R., Cooper, G., Odell, G., Voytek, B. y Whitman, A. (1974). Experimentation in the formation of edge damage: A new approach to lithic analysis. Journal of Field Archaeology, 1(1-2), 171-196.

» Yacobaccio, H., Catá, P., Solá, P. y Alonso, S. (2008). Estudio arqueológico y fisicoquímico de pinturas rupestres en Hornillos 2 (Puna de Jujuy). Estudios Atacameños, 36, 5-28.

" Yacobaccio, H. y Morales, M. (2011). Ambientes pleistocénicos y ocupación humana temprana en la Puna Argentina. Boletín de Arqueología PUCP, 15, 337-356. 